\title{
A Bufferable Tuned-Mass Damper of an Offshore Platform against Stroke and Response Delay Problems under Earthquake Loads
}

\author{
Qiong Wu, ${ }^{1}$ Xilu Zhao, ${ }^{1}$ Shuai He, ${ }^{1}$ Wenxian Tang, ${ }^{2}$ and Rencheng Zheng ${ }^{3}$ \\ ${ }^{1}$ College of Mechanical Engineering, Saitama Institute of Technology, Saitama 369-0293, Japan \\ ${ }^{2}$ Jiangsu University of Science and Technology, Jiangsu 2212003, China \\ ${ }^{3}$ Institute of Industrial Science, The University of Tokyo, Tokyo 153-8505, Japan \\ Correspondence should be addressed to Rencheng Zheng; topzrc@iis.u-tokyo.ac.jp
}

Received 28 July 2016; Accepted 30 October 2016

Academic Editor: Alba Sofi

Copyright (C) 2016 Qiong Wu et al. This is an open access article distributed under the Creative Commons Attribution License, which permits unrestricted use, distribution, and reproduction in any medium, provided the original work is properly cited.

\begin{abstract}
A tuned-mass damper (TMD) is applied to ensure the safety and stability of an offshore platform in practice; however, damper stroke and response delay problems always result in intractable performances of vibration control while exposed to large earthquake loads. Therefore, this paper proposes a bufferable TMD, a passive TMD with buffers on both sides, to improve the performance of offshore platforms subjected to large seismic waves. A comprehensive simulation and experimental study was executed to investigate the dynamic performances of the bufferable TMD, by application of a 1:200-scale offshore platform prototype. It is verified that the bufferable TMD can be effective in absorbing the stroke energy, while the damper exceeds limitations of motion. Meanwhile, the bufferable TMD can maintain high-response characteristics. In conclusion, the experimental results indicate that the displacement, acceleration, and frequency performances of an offshore platform can be significantly decreased, and the evaluation indices show that the method is effective in reducing overall vibration levels and maximum peak values, with the application of the bufferable damper system.
\end{abstract}

\section{Introduction}

As one of the typical offshore structures, offshore jacket platform plays an indispensable role in the development and utilization of marine resources [1]. Located in hostile ocean environments, offshore platforms are exposed to external disturbances such as winds and earthquakes, which generally lead to large oscillation of the system [2]. The vibrations not only gradually damage the platform structural system but also cause the problem of uncomfortable environment of staying. Therefore, many efforts have been made to control the vibration of the structures, to increase the stability of the structure, and to ensure the safety of the offshore platforms.

However, there are two intractable problems while taking into account the possibility of damage of the offshore platform during a high-intensity earthquake. One such problem is that the large earthquake excitation may cause local damage to the vibration absorber; therefore, it does not exhibit enough vibration control performance in case of large earthquakes. Another problem is that the duration of an earthquake excitation event is generally short, and the maximum deformational influence on a platform mainly occurs from its initial few seconds. However, rare attention is being paid to resolve the two unavoidable problems in the same time.

To solve the problem of stroke between vibration absorber and target structure, several attempts had been made to decrease the stroke, by introducing the idea of impact dampers. An impact damper is a freely moving mass, constrained by stops fixed to a dynamically excited structure to be controlled. The impact damper results from the exchange of momentum during impacts between the mass and the stops while the structure vibrates. Energy is dissipated as heat and noise together with the development of high-frequency vibrations in the structure [3]. On the other hand, the impact dampers will produce impulsive loads between the 
two coupled systems and will cause a high-level noise during the impact process. Meanwhile, large contact forces will be produced and result in material deterioration and local deformation accompanying plastic collisions. In contrast, buffer materials have been incorporated in the impact damper system to reduce impact forces [4]. The dissipative properties of granular materials had been analytically studied using the particle dynamic method, and how the analysis of energy loss rate displays different damping regimes in the amplitudefrequency plane of the excitation force was demonstrated [5]. Recently, vibration control technologies, implemented based on platform type, had achieved significant success in mitigating the vibration of land-based structures. Zhang et al. [6] proposed a network-based modeling and event-triggered $H \infty$ reliable control for an offshore structure. It can suppress the vibration of the offshore structure to almost the same level as the $H 1$ controller, while the former requires less control cost. Specifically, a more general uncertain dynamic model of the offshore platform was developed, and then a novel delayed sliding mode control scheme using mixed current and delayed states was proposed in [7]. It is shown through the simulating results that this scheme is more effective in both improving the control performance and reducing control force of the offshore platform. Chen et al. [8] performed numerical modeling of impact damping with a discrete element method, and it indicated that the collision and friction mechanism might play different or equivalent roles in energy dissipation, under different vibration and particle system parameters. In addition, most of the numerical simulations were based on the discrete element method, which was initially proposed by Cundall and Strack [9].

As to the fundamental theory of impact dampers, numerical and experimental studies had been undertaken to test specific applications, check theoretical results, and select parameters of impact damper systems [10-13]. An impact damper was used to control a simple degree-of-freedom system under free and sinusoidal allied forced vibrations, with an optimum damping effect by adjusting the mass ratio and clearance. Li and Darby [14] and Liu et al. [15] conducted a series of experimental investigations to examine the effect of an impact damper, using equivalent viscous damping model to represent the nonlinearity. Damping characteristics of an impact damper were also studied to improve the damping capability. Fang and Tang [16] developed an improved analytical model using multiphase flow theory based on the previous work of Wu et al. [17]. Recently, experimental work has studied the effect of controlling friction-driven oscillations using an impact damper, and the effects of mass ratio, coefficient of restitution, and clearance on the performance of an impact damper were verified through the experimental and numerical investigations [18]. The above studies had mentioned that a vibration controller with damper can provide a viable and efficient way to attenuate vibrations; however, it is still not discussed for the response delay problem of the controller with damper, which may result in undesirable influence for vibration control during the initiation of seismic motion.

For an earthquake excitation in which its duration is substantially shorter, considerable disasters often occurred during the initial period of an earthquake load. The several previous studies [19-21] used the vibration absorber with viscous damping to improve the vibration control effectiveness; however, because the high-response performance of the dampers is not considered, the dampers may not have enough reaction time to produce a significant effect in the initial seconds of the earthquake excitation. Tsai [22] simulated a tuned-mass damper (TMD) with a range of parameters under different earthquake excitations. A TMD with damping was found to have little reaction to the structural response in the initial seconds following the occurrence of an earthquake, and, therefore a virtual accelerator was proposed to improve the TMD performance. Recently, a passive TMD without damper was proposed to overcome the earthquake excitation during the first 3 seconds; however, the spring links between the TMD and the target structures were not considered for the case of large earthquakes [23]. The duration of an earthquake excitation is generally short, and the maximum influence on a platform's deformation mainly results from its initial seconds. Therefore, it is critical that the high-response speed of the TMD occurs within the initial seconds of the excitation. To overcome this problem, this study proposed a passive TMD without damper for improving the highresponse performance under critical earthquake loads.

Furthermore, the above-mentioned studies for the traditional TMD did not simultaneously consider the stroke and response delay problems. On the one hand, even though the TMD with dampers may be acceptable for energy dissipation accompanied with large seismic motions, it may become questionable for its low-response performance. On the other hand, although the TMD without damper can enhance the high-response performance, it may not avoid the collision destruction under intense seismic waves. Therefore, this paper will propose a bufferable TMD, which can combine the advantages of the impact damper with buffer and highresponse damper against stroke and response delay problems. This paper proposes a bufferable tuned-mass damper of an offshore platform against stroke energy and response delay problems under seismic waves. Both simulation and experimentation studies are proposed to verify the effectiveness and advantages of the proposed methods.

This paper involves experimental and analytical investigations that comprehensively extend the understanding of the high-response performance and damping characteristics of bufferable TMD systems under two large seismic vibrations. The experimental processes are based on a prototype of an existing offshore jacket platform, and the bufferable, highresponse, absorption characteristics are discussed dependent on the results of the amplitude and frequency responses.

\section{Methods}

2.1. Description. A jack-up offshore platform comprises a rectangular platform resting on four independent operating legs, with a TMD attached beneath the platform. There are two normal types of passive TMD: those with and without dampers (Figure 1). The TMD without damper has a high-response performance, and the TMD with damper can dissipate energy owing to the damping characteristics [23]. 


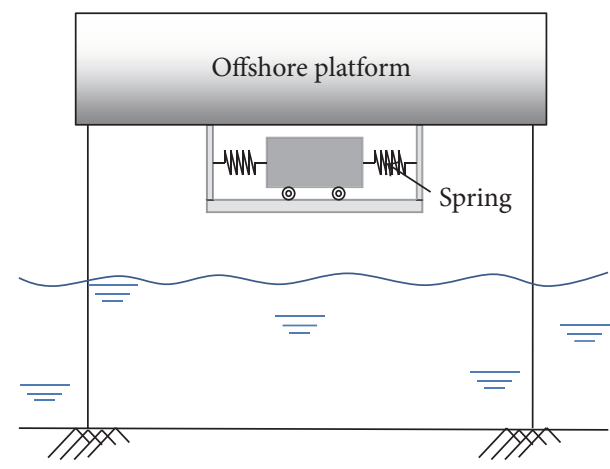

(a) TMD without damper

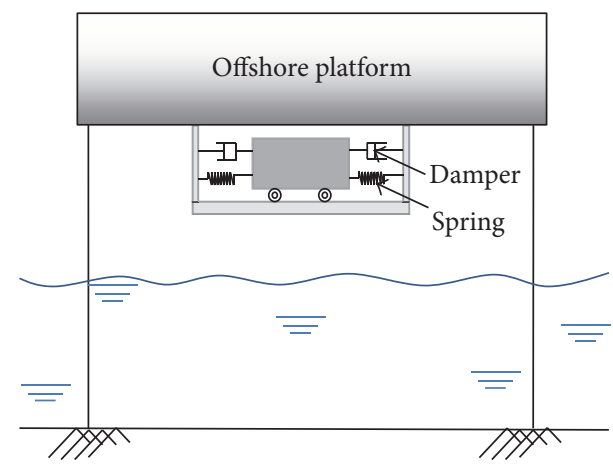

(b) TMD with damper

FIGURE 1: Schematic of a jack-up offshore platform with TMD without damper (a) and TMD with damper (b).

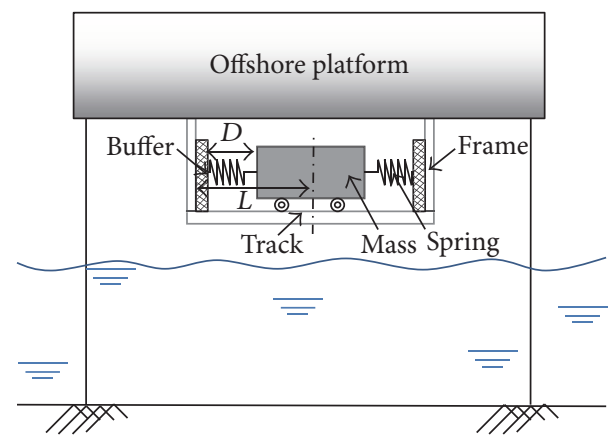

FIGURE 2: Schematic of a jack-up offshore platform with a bufferable TMD.

This study makes use of a TMD with a buffer to realize the advantages of both the TMD with and without damper. The TMD device (Figure 2) consists of a frame, a mass, two springs, four wheels, two tracks, and two buffers. The buffers, which adopt buffering materials, can be fixed to the frame by adhesive. The distance between the buffer and mass can be adjusted depending on performance requirements.

2.2. Modeling. As presented in Figure 3, a systemic model of a jack-up offshore platform with a bufferable TMD can be considered as a generalized structure consisting of the main structure of the offshore platform and the substructure of the TMD. The buffers are modeled by two linear contact springs and dampers, as $k_{B}$ and $c_{B}$, respectively.

The motion of the system can be treated as a piecewise linear process. $x_{1}$ and $x_{2}$ are the displacement of main structure and substructures, respectively. When $\left|x_{2}-x_{1}\right| \leq d$, the mass can move without collisions with the buffers, where $d$ is the distance between the buffer and mass of the TMD, as shown in Figure 1; therefore, the dynamic model can be expressed by normal forms:

$$
\begin{array}{r}
m_{1} \ddot{x}_{1}+c_{1} \dot{x}_{1}+k_{1} x_{1}-k_{2}\left(x_{2}-x_{1}\right)=-m_{1} \ddot{x}_{V}, \\
m_{2} \ddot{x}_{2}+k_{2}\left(x_{2}-x_{1}\right)=-m_{2} \ddot{x}_{V},
\end{array}
$$

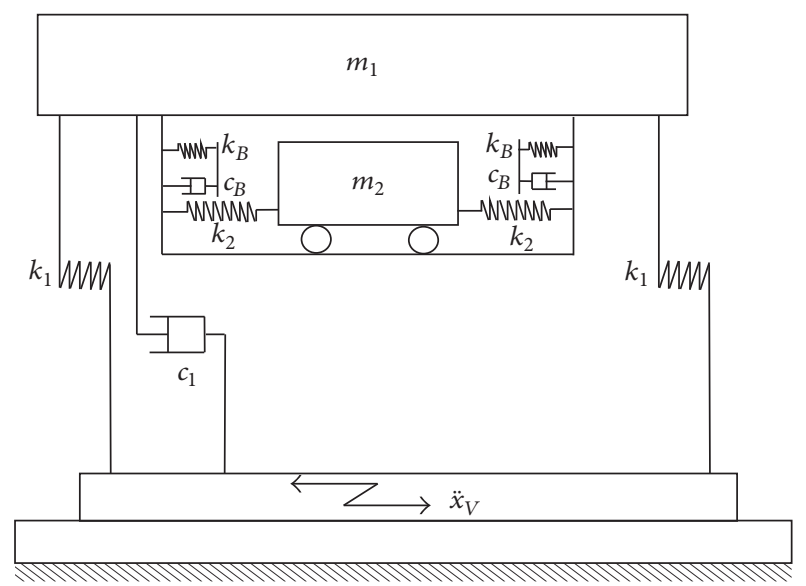

FIGURE 3: Systemic model of a jack-up offshore platform with a bufferable TMD.

where $m_{1}, c_{1}$, and $k_{1}$ are the mass, damping, and stiffness of the main structure, respectively; $m_{2}$ and $k_{2}$ are the mass and stiffness of the substructure, respectively; $\ddot{x}_{V}$ is the acceleration vector of the seismic loads on the main structure.

When $\left|x_{2}-x_{1}\right|>d$, the mass collides with the left or right buffers; therefore, the dynamic model can be expressed as

$$
\begin{aligned}
& m_{1} \ddot{x}_{1}+c_{1} \dot{x}_{1}-c_{B}\left(\dot{x}_{2}-\dot{x}_{1}\right)+k_{1} x_{1}-k_{2}\left(x_{2}-x_{1}\right) \\
& -k_{B}\left(\left|x_{2}-x_{1}\right|-d\right) \operatorname{sgn}\left(x_{2}-x_{1}\right)=-m_{1} \ddot{x}_{V}, \\
& m_{2} \ddot{x}_{2}+c_{B}\left(\dot{x}_{2}-\dot{x}_{1}\right)+k_{2}\left(x_{2}-x_{1}\right) \\
& \quad+k_{B}\left(\left|x_{2}-x_{1}\right|-d\right) \operatorname{sgn}\left(x_{2}-x_{1}\right)=-m_{2} \ddot{x}_{V},
\end{aligned}
$$

where $m_{1}, c_{1}$, and $k_{1}$ are the mass, damping, and stiffness of the main structure, respectively; $m_{2}$ and $k_{2}$ are the mass and stiffness of the substructure, respectively; $k_{B}$ and $c_{B}$ are the stiffness and damping of buffers, respectively; $x_{1}$ and $x_{2}$ are the displacement of main structure and substructures, respectively; $\ddot{x}_{V}$ is the acceleration vector of the seismic loads on the main structure. 
The central difference method [24] is used for numerically solving the above equations. A time marching scheme for the forward difference method is used, where $x^{(i)}=x\left(t=t_{i}\right)$ and the time interval $\Delta t=t_{i+1}-t_{i}$. The differential acceleration and velocity can be expressed as

$$
\begin{aligned}
& \ddot{x}^{(i)}=\frac{x^{(i+1)}-2 x^{(i)}+x^{(i-1)}}{\Delta^{2} t}, \\
& x_{1}{ }^{(i+1)}=\frac{\left(2 m_{1}-k_{1} \Delta^{2} t-k_{2} \Delta^{2} t\right) x_{1}{ }^{(i)}+k_{2} \Delta^{2} t x_{2}{ }^{(i)}+\left(-m_{1}+0.5 c_{1} \Delta t\right) x_{1}{ }^{(i-1)}-m_{1} \ddot{x}_{V} \Delta^{2} t}{m_{1}+0.5 c_{1} \Delta t} \\
& x_{2}{ }^{(i+1)}=\frac{\left(2 m_{2}-k_{2} \Delta^{2} t\right) x_{2}{ }^{(i)}+k_{2} \Delta^{2} t x_{1}{ }^{(i)}-m_{2} x_{2}{ }^{(i-1)}-m_{2} \ddot{x}_{V} \Delta^{2} t}{m_{2}} .
\end{aligned}
$$

Equations (3) can be substituted into (1) and (2) to calculate the displacement difference. When $\left|x_{2}-x_{1}\right| \leq d$,
When $\left|x_{2}-x_{1}\right|>d$, for the case of $x_{2}>x_{1}$, the formula can be expressed as

$$
\begin{aligned}
& x_{1}{ }^{(i+1)}=\frac{A k_{22}-B k_{12}}{k_{11} k_{22}-k_{21} k_{12}}, \\
& x_{2}{ }^{(i+1)}=\frac{B k_{22}-A k_{12}}{k_{11} k_{22}-k_{21} k_{12}},
\end{aligned}
$$

where

$$
\begin{aligned}
A= & -\left(2 m_{1}+k_{1} \Delta^{2} t+k_{2} \Delta^{2} t+k_{b} \Delta^{2} t\right) x_{1}{ }^{(i)} \\
& -\left(-k_{2} \Delta^{2} t-k_{b} \Delta^{2} t\right) x_{2}{ }^{(i)} \\
& -\left(m_{1}-0.5 c_{1} \Delta t-0.5 c_{b} \Delta t\right) x_{1}{ }^{(i-1)} \\
& -0.5 c_{b} \Delta t x_{2}{ }^{(i-1)}-k_{b} \Delta^{2} t \cdot d^{2}-m_{1} \ddot{x}_{V}{ }^{2} \Delta^{2} t, \\
B= & -\left(-k_{2} \Delta^{2} t-k_{b} \Delta^{2} t\right) x_{1}{ }^{(i)} \\
& -\left(-2 m_{2}+k_{2} \Delta^{2} t+k_{b} \Delta^{2} t\right) x_{2}{ }^{(i)} \\
& -0.5 c_{b} \Delta t x_{1}{ }^{(i-1)}-m_{2} x_{2}{ }^{(i-1)}+k_{b} \Delta^{2} t \cdot d \\
& -m_{2} \ddot{x}_{V} \Delta^{2} t \\
k_{11}= & m_{1}+0.5 c_{1} \Delta t \\
k_{12}= & -0.5 c_{b} \Delta t \\
k_{21}= & -0.5 c_{b} \Delta t \\
k_{22}= & m_{2}+0.5 c_{b} \Delta t .
\end{aligned}
$$

When $\left|x_{2}-x_{1}\right|>d$, for the case of $x_{2}<x_{1}$, the formula can be expressed as

$$
\begin{aligned}
& x_{1}{ }^{(i+1)}=\frac{A^{\prime} k_{22}-B^{\prime} k_{12}}{k_{11}^{\prime} k_{22}-k_{21}^{\prime} k_{12}}, \\
& x_{2}{ }^{(i+1)}=\frac{B^{\prime} k_{22}-A^{\prime} k_{12}}{k_{11} k_{22}-k_{21} k_{12}},
\end{aligned}
$$

where

$$
\begin{aligned}
A^{\prime}= & -\left(2 m_{1}+k_{1} \Delta^{2} t+k_{2} \Delta^{2} t+k_{b} \Delta^{2} t\right) x_{1}{ }^{(i)} \\
& -\left(-k_{2} \Delta^{2} t-k_{b} \Delta^{2} t\right) x_{2}{ }^{(i)} \\
& -\left(m_{1}-0.5 c_{1} \Delta t-0.5 c_{b} \Delta t\right) x_{1}{ }^{(i-1)} \\
& -0.5 c_{b} \Delta t x_{2}{ }^{(i-1)}+k_{b} \Delta^{2} t \cdot d^{2}-m_{1} \ddot{x}_{V}{ }^{2} \Delta^{2} t \\
B^{\prime}= & -\left(-k_{2} \Delta^{2} t-k_{b} \Delta^{2} t\right) x_{1}{ }^{(i)} \\
& -\left(-2 m_{2}+k_{2} \Delta^{2} t+k_{b} \Delta^{2} t\right) x_{2}{ }^{(i)} \\
& -0.5 c_{b} \Delta t x_{1}{ }^{(i-1)}-\left(m_{2}-0.5 c_{b} \Delta t\right) x_{2}{ }^{(i-1)} \\
& -k_{b} \Delta^{2} t \cdot d-m_{2} \ddot{x}_{V} \Delta^{2} t .
\end{aligned}
$$




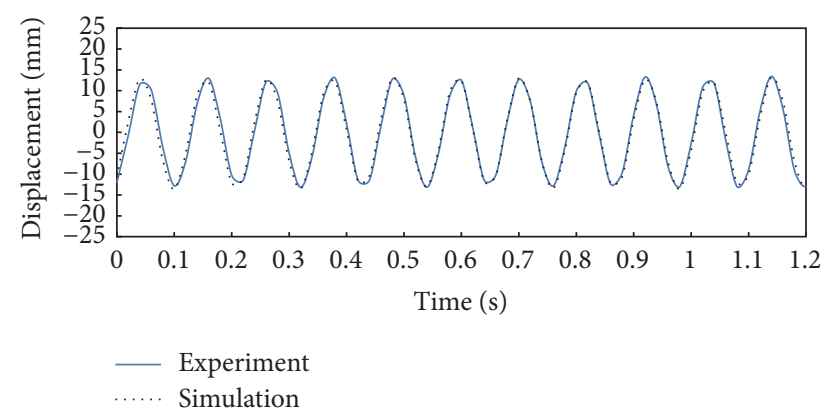

FIGURE 4: Displacements of the offshore platform with the bufferable TMD under sinusoidal excitation.

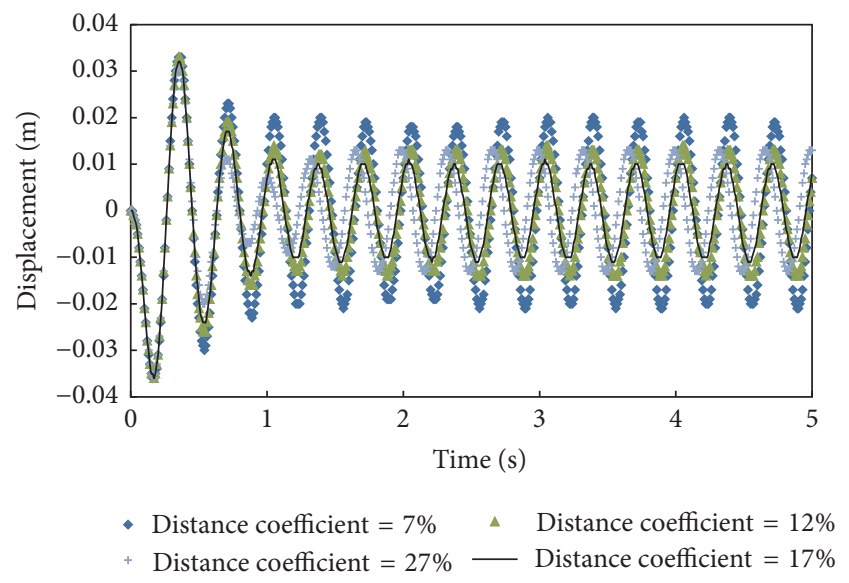

FIGURE 5: Displacement responses of the modeled offshore platform corresponding to various distance coefficients under sinusoidal waves.

represented as sinusoidal excitation (Figure 4). The theoretical response is observed to be identical to the experimental response over the entire time period, which suggests that the analytical method can yield estimates of the damper system response under earthquake excitations with an acceptable accuracy.

\subsection{Parameter Identification}

2.3.1. Parameters under Sinusoidal Excitation. A distance coefficient $\bar{D}$ is used to discuss the optimal distance based on its influence on the maximum vibration reduction of peak values under either sinusoidal or seismic waves. The distance coefficient is defined as $\bar{D}=d / L$, where $d$ is the distance between the buffer and mass and $L$ is half of the total length of the TMD equipment.

Initially, a numerical simulation is carried out for different distance coefficients under a sinusoidal wave. The sinusoidal frequency is $3 \mathrm{~Hz}$, corresponding to the resonance frequency of the main system, which is also $3 \mathrm{~Hz}$. The mass ratio of the main structure and substructure is 0.3 , and damping coefficient of the buffer is 0.012 . The input excitation, mass ratio, and damping coefficient are kept constant while the distance between the buffer and mass of the TMD is varied for parameter identification.

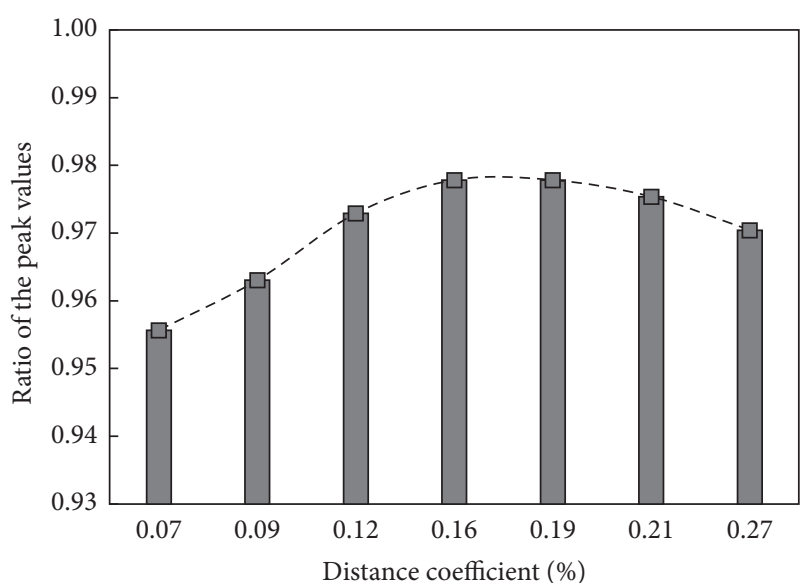

FIGURE 6: Ratios of the peak values of the modeled offshore platform with and without bufferable TMD for various distance coefficients under sinusoidal waves.

In Figure 5, displacements of the main system with the bufferable TMD are denoted by the dash-dotted and solid lines for various distances. When the distance coefficient is $17 \%$, the displacement responses of the offshore platform are lowest.

The ratios of the peak values of the offshore platforms with and without bufferable TMD are calculated for the different distance coefficients (Figure 6). When the distance coefficient is $16 \%$ to $19 \%$, the ratios of the peak values are highest, which indicates that its performance for vibration absorption is better than at other distance coefficients.

2.3.2. Parameters under Seismic Excitation. For further consideration of the influence of the distance between the buffer and mass of the TMD under seismic excitation, numerical simulations of the Fukujima NS and Taft EW seismic waves were undertaken. Fukujima NS refers to the NS component recorded in Japan on March 11, 2011. The corresponding earthquake's magnitude was 9.0 with peak acceleration of $341 \mathrm{~cm} / \mathrm{s}^{2}$. Taft EW refers to the EW component recorded in Kern County, CA, USA, on July 21, 1952. The corresponding earthquake had a magnitude of 7.7 and a peak acceleration value of $175.9 \mathrm{~cm} / \mathrm{s}^{2}$.

As shown in Figure 7(a), the ratios of the peak values between the cases with and without the bufferable TMD were calculated for various distances. When the distance coefficient is $17 \%$ to $19 \%$, the ratios of the peak values become higher, and their performance is better than at other distance coefficients. Figure 7(b) shows that the offshore platform can achieve better vibration suppression when the distance coefficient is around $16 \%$.

\section{Experiment}

3.1. Setup. As shown in Figure 8, the testing system comprised a computer, vibration signal generator, amplifier, shaker, offshore platform system, acceleration sensor, laser displacement sensor, and fast Fourier transform analyser. 


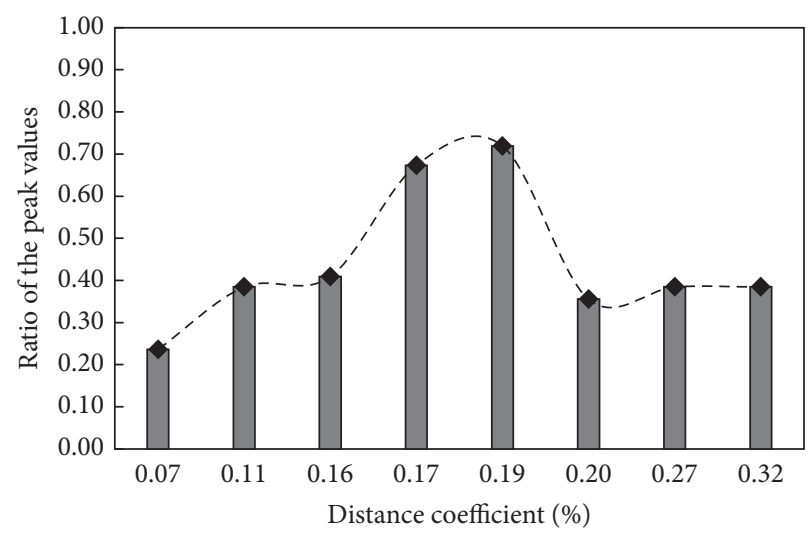

(a) Ratio of the peak values under Taft EW seismic wave

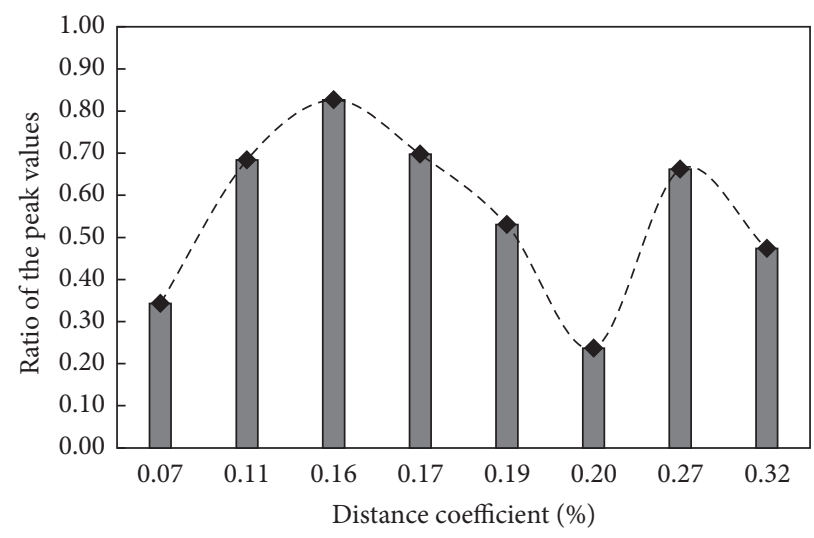

(b) Ratio of the peak values under Fukujima NS seismic wave

FIGURE 7: Ratios of the peak values for the offshore platform with and without bufferable TMD for different distance coefficients under (a) the Taft EW seismic waves and (b) the Fukujima NS.

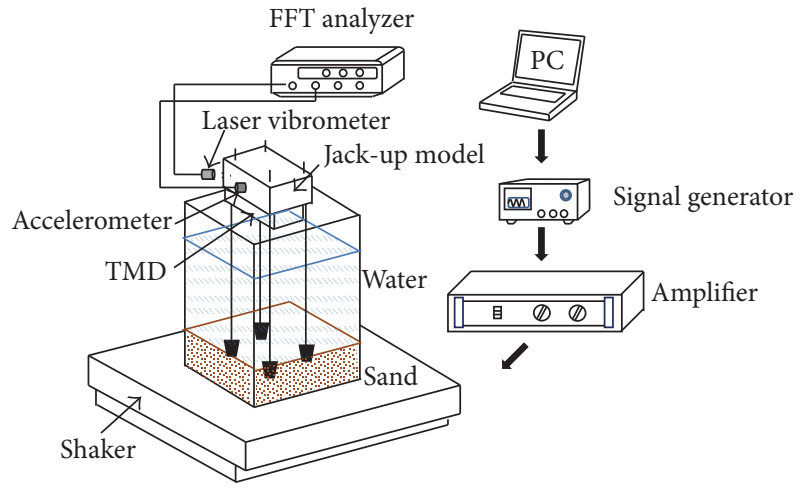

FIGURE 8: Diagram of the testing system (PC: personal computer, TMD: bufferable tuned-mass damper, and FFT Analyser: fast Fourier transform analyser).

The Bohai number 5 offshore jack-up platform, located in the Southern Sea, was used as a research target to analyse the practical effectiveness of a TMD. A 1:200-scale model of the actual four-column platform was constructed. The size of the working platform is $57.5 \times 34.0 \times 5.50 \mathrm{~m}$, with operating leg lengths of $78 \mathrm{~m}$ and diameters of $3 \mathrm{~m}$. The minimum operating water depth is $4 \mathrm{~m}$.

The modeled operating platform was scaled and simplified into a horizontal rectangular metal mass with support columns constructed of hollow tubes. Cylindrical pile shoes were set at the base of each column and the connections between the columns and the operating platform were rigid. The model platform was placed in a tank that was fixed to the shaker apparatus capable of simulating seismic loads. The response of the structure was measured using an accelerometer and a laser vibrometer.

In the experiment, the sand height was $80 \mathrm{~mm}$ and the water depth was $400 \mathrm{~mm}$. The mass of the main structure of the model platform was $2.346 \mathrm{~kg}$ and the mass of the bufferable TMD was $0.591 \mathrm{~kg}$. Through experimental validation, the damping coefficient of the offshore platform was determined to be 0.012 , with a buffer damping coefficient of 0.012 .

3.2. Processes. Initially, the substructure of the bufferable TMD was installed at the bottom of the model platform to measure the first natural frequency of the complete structure. The input signal was a $0-8 \mathrm{~Hz}$ sweep signal. The peak frequency response function for the platform was centered at $3 \mathrm{~Hz}$.

The second step was to remove the bufferable TMD from the platform and to attach it to the shaker. Then, the spring stiffness of the bufferable TMD was adjusted to make the first natural frequency of the bufferable TMD the same as that of the platform.

In the next step, two types of seismic wave (Fukujima NS and Taft EW) were generated by the signal generator and fed to the shaker to evaluate the effectiveness of the vibration control system. The recording time was $25 \mathrm{~s}$ and $30 \mathrm{~s}$, respectively, and the sampling frequency was $50 \mathrm{~Hz}$ for the two seismic waves. Consequently, the displacement and acceleration of the offshore platform and the relative displacement between the offshore platform and the bufferable TMD were recorded.

\section{Results}

4.1. Amplitude Response Analysis. As presented in Figure 9, the time series of the responses are shown for the main structure with and without the bufferable TMD under the excitations of the Fukujima NS and Taft EW seismic waves. The experimental results show that the peak values of the displacement responses decreased significantly with bufferable TMD control, when compared with those cases without bufferable TMD control. It can be observed that this decreasing tendency was more significant for the acceleration responses. These results indicate that the control performance of the bufferable TMD is effective as an energy dissipation device for the reduction of the main structural response.

It is particularly important for vibration suppression under excitation by seismic waves that the bufferable TMD 


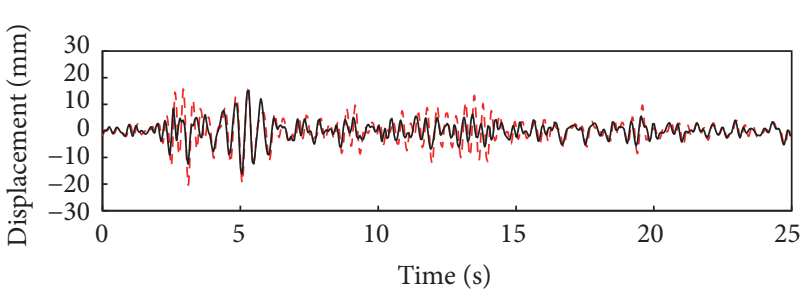

- - No TMD control

— With TMD control

(a) Displacement results for the Fukujima NS seismic wave

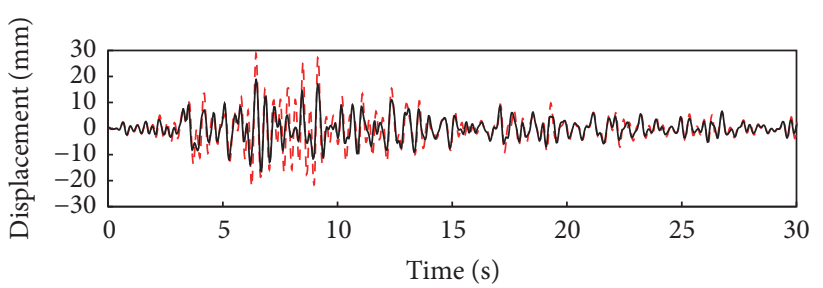

$\begin{array}{ll}--- & \text { No TMD control } \\ - & \text { With TMD control }\end{array}$

(c) Displacement results for the Taft EW seismic wave

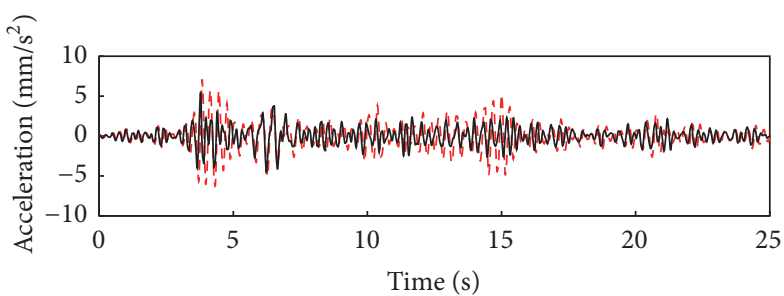

- - No TMD control

— With TMD control

(b) Acceleration results for the Fukujima NS seismic wave

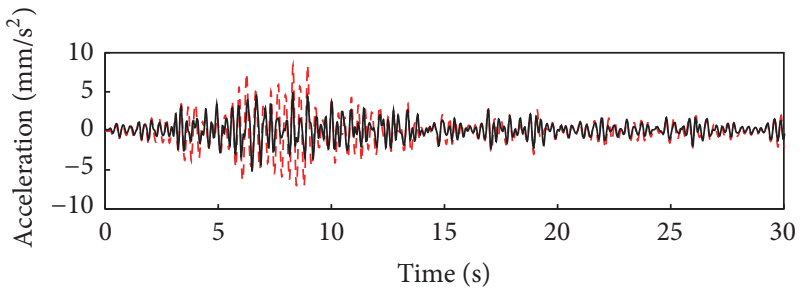

- - No TMD control

— With TMD control

(d) Acceleration results for the Taft EW seismic wave

FIGURE 9: Amplitude responses of the platform subjected to the Fukujima NS and Taft EW seismic waves: (a) displacement results for Fukujima NS, (b) acceleration results for Fukujima NS, (c) displacement results for Taft EW, and (d) acceleration results for Taft EW.

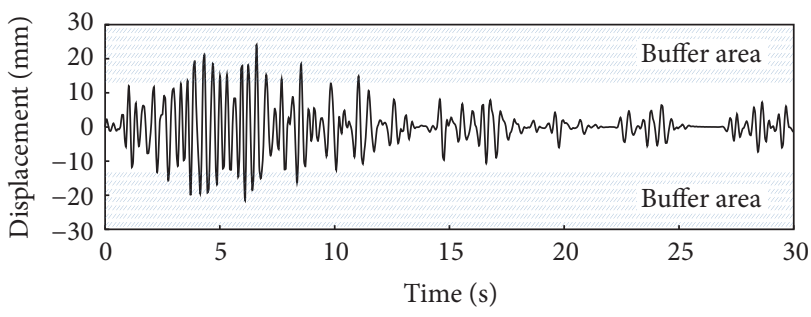

(a) Displacement of the mass under Taft EW seismic waves

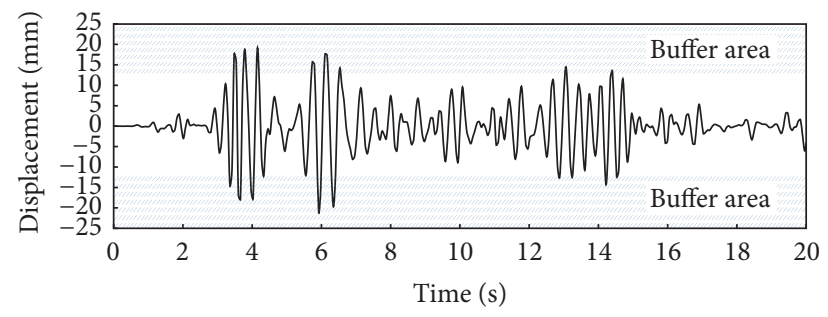

(b) Displacement of the mass under Fukujima NS seismic waves

Figure 10: Amplitude responses of the mass of the bufferable TMD under Fukujima NS and Taft EW seismic waves: (a) displacement result under Taft EW and (b) displacement result under Fukujima NS.

can effectively reduce relatively high-amplitude displacements and accelerations. These high-amplitude displacements occurred during the early period of the test and the bufferable TMD responded quickly to these early vibration excitations. For the relatively low-amplitude displacements and accelerations, the bufferable TMD control resulted in little effective reduction; however, these low-amplitude local effects provided only a small contribution to overall platform vibration.

In this experiment, the studies focus on the distances at which the maximum effective damping is observed. Thereby, the distance coefficient is $17 \%$ and the distance is $13 \mathrm{~mm}$. Note that the impacts between the mass and buffers always occur at the time at which the primary system has reached its maximum displacement. The vibration control effectiveness of a bufferable absorber comes from collisions of the mass with the buffer, and results in an exchange of momentum.

As shown in Figure 10, as the mass is displaced into the buffer areas, there are collisions between the mass and the buffers. This means that significant energy is absorbed during the collision owing to an exchange of momentum. Greater impulse momentum can more efficiently result in a decreasing response velocity for the offshore platform.

4.2. Frequency Response Analysis. Based on a frequency response analysis, the power spectral density (PSD) results are presented in Figures 11 and 12 for the displacement and acceleration of the platform with and without the bufferable TMD. The maximum amplitude of the PSD is observed to occur at around $3 \mathrm{~Hz}$ for the case without bufferable TMD control; the resonance reaction of the platform is seen to occur around this frequency. This explains why the designed bufferable TMD's frequency was nearly $3 \mathrm{~Hz}$.

Therefore, the use of bufferable TMD controls is significantly effective in reducing the vibration response of the main structure; in particular, the peak responses are reduced considerably around frequencies of $3 \mathrm{~Hz}$. The overall vibration response can be decreased significantly by reducing 


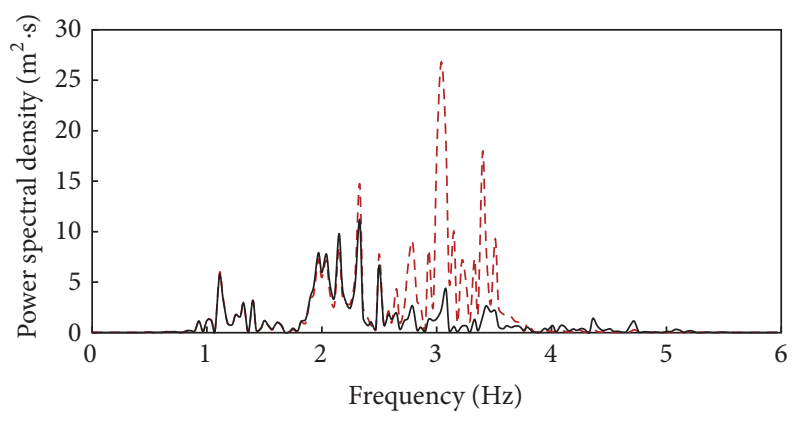

- - No TMD control

- With TMD control

(a) Power spectral density of displacement for the Fukujima NS seismic waves

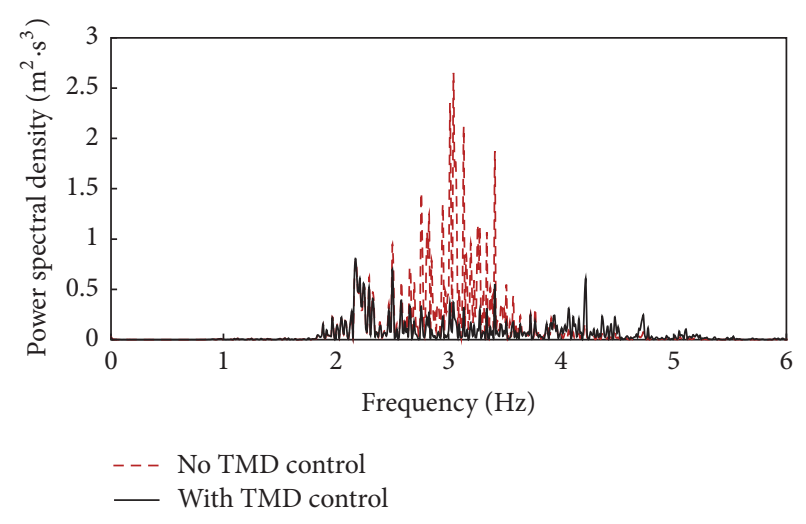

(b) Power spectral density of acceleration for the Fukujima NS seismic waves

FIGURE 11: Power spectral density of the displacement of the platform with and without bufferable TMD, under the Fukujima NS seismic waves (a) and the Taft EW seismic waves (b).

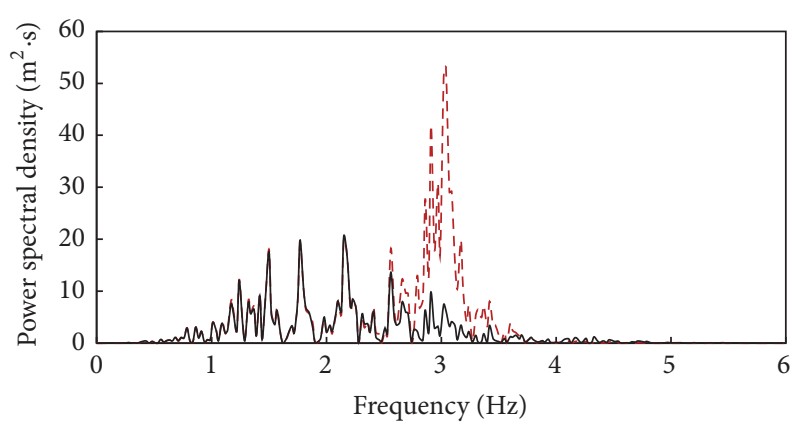

- - No TMD control

(a) Power spectral density of displacement for the Taft EW seismic waves

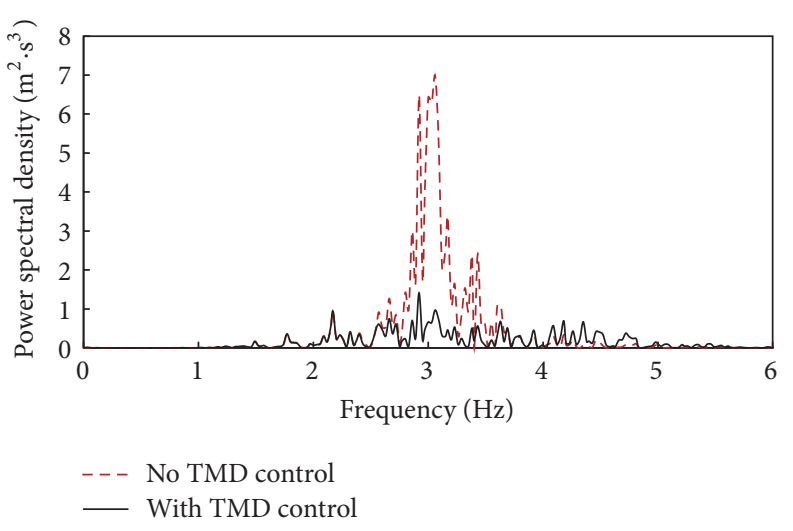

(b) Power spectral density of acceleration for the Taft EW seismic waves

FIGURE 12: Power spectral density of the accelerations of the platform with and without bufferable TMD, under the Fukujima NS seismic waves (a) and the Taft EW seismic waves (b).

first-mode vibrations, and the resulting PSD curves explain why the time series response is effective for vibration reduction. Consequently, a single damper tuned to the fundamental mode is adequate for reducing structural vibrations resulting from earthquake excitations. Investigations of frequency bands above or below the fundamental frequency revealed no negative effects at nondominant frequencies.

4.3. Evaluation Index. To quantify the control performance, the root mean square (RMS) of the displacement or acceleration responses is adopted for the platform:

$$
\mathrm{RMS}=\sqrt{\frac{1}{n} \sum_{i=1}^{n} y_{i}^{2}},
$$

where $y_{i}$ is the sampling value of the displacement or acceleration of the platform as it responds to seismic waves.
To evaluate the effectiveness of vibration reduction during the entire earthquake period, a relative index was applied as

$$
J=\frac{\mathrm{RMS}_{\mathrm{ctrl}}}{\mathrm{RMS}_{\text {unctrl }}}
$$

where $\mathrm{RMS}_{\text {ctrl }}$ and $\mathrm{RMS}$ unctrl are the RMS values of the displacement or acceleration responses of the main structure for the entire earthquake period, for the cases with and without a bufferable TMD system, respectively.

To evaluate the maximum response reduction, a maximum peak value was selected as $y_{\max }=\max \left\{y_{i}\right\}$. Then, a relative ratio of the maximum peak values can be obtained as

$$
\beta=\frac{y_{\text {max-unctrl }}-y_{\text {max-ctrl }}}{y_{\text {max }- \text { unctrl }}},
$$

where $y_{\text {max-ctrl }}$ and $y_{\text {max-unctrl }}$ are the maximum peak values of the displacement or acceleration response of the platform with and without a bufferable TMD system, respectively. 


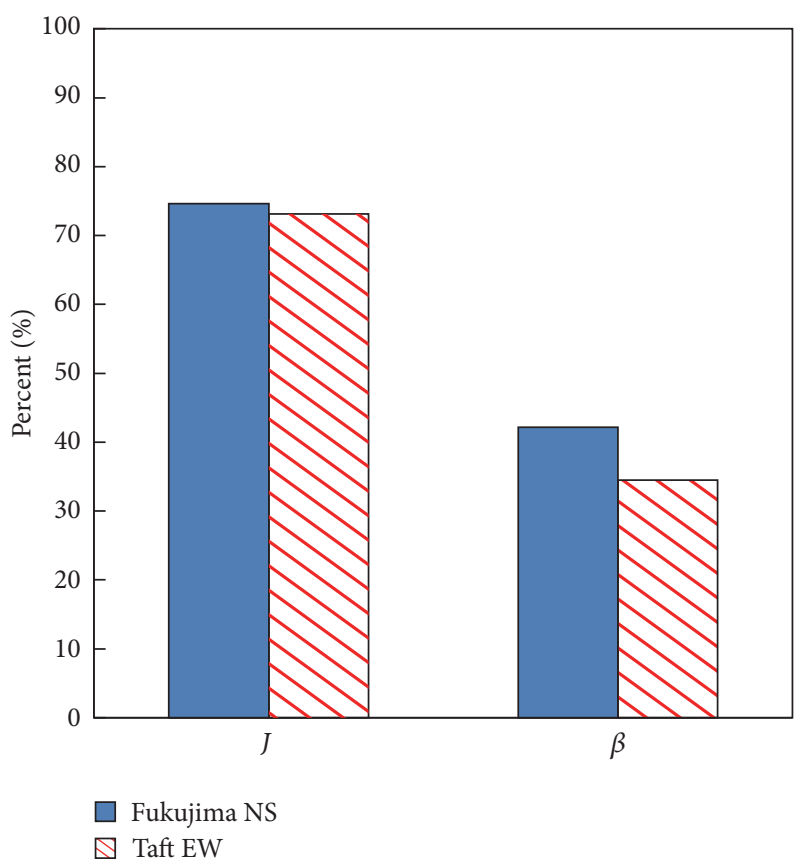

(a) Evaluation indices of the displacement results

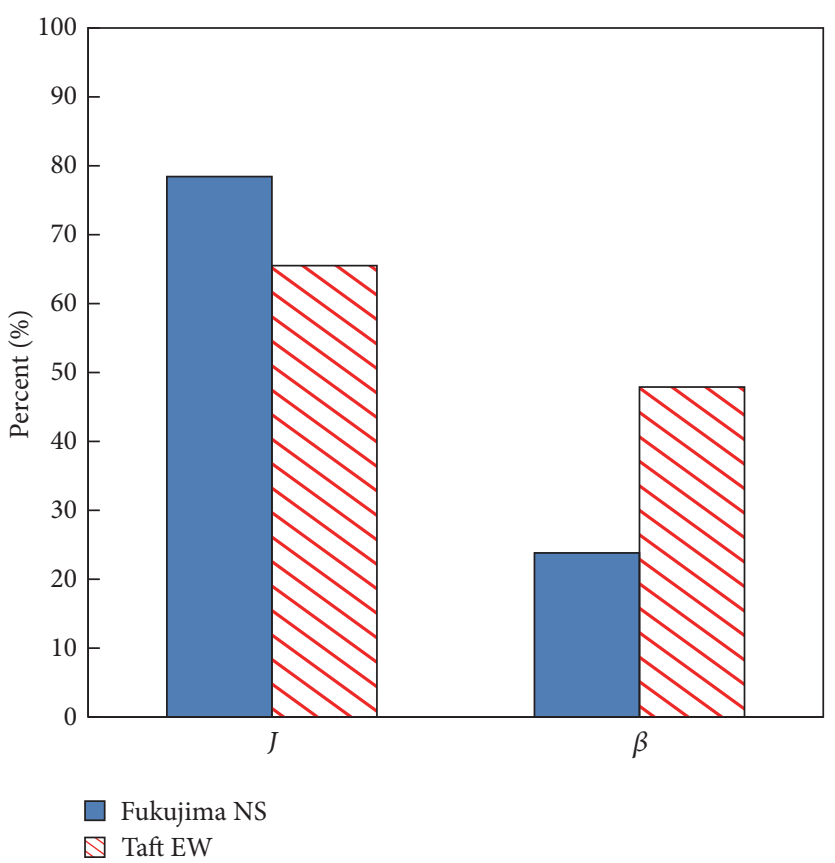

(b) Evaluation indices of the acceleration results

FIGURE 13: Experimental results for the evaluation parameters under Fukujima NS and Taft EW conditions: (a) evaluation indices of the displacement results and (b) evaluation indices of the acceleration results.

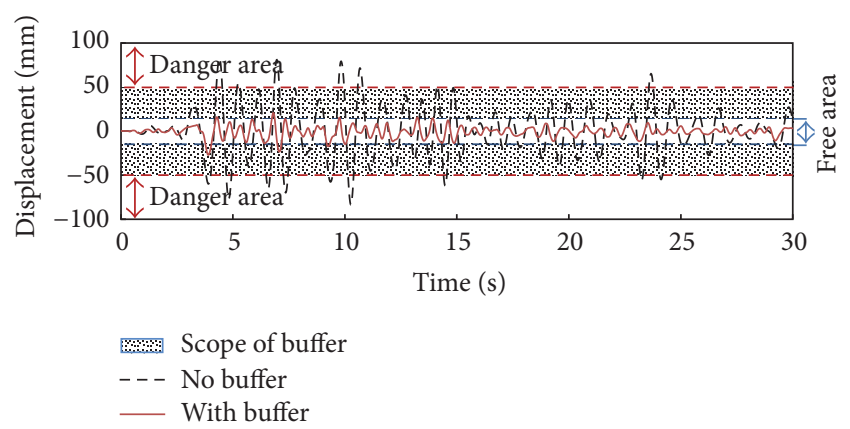

(a) Relative displacement results for a TMD mass with a Taft EW seismic wave

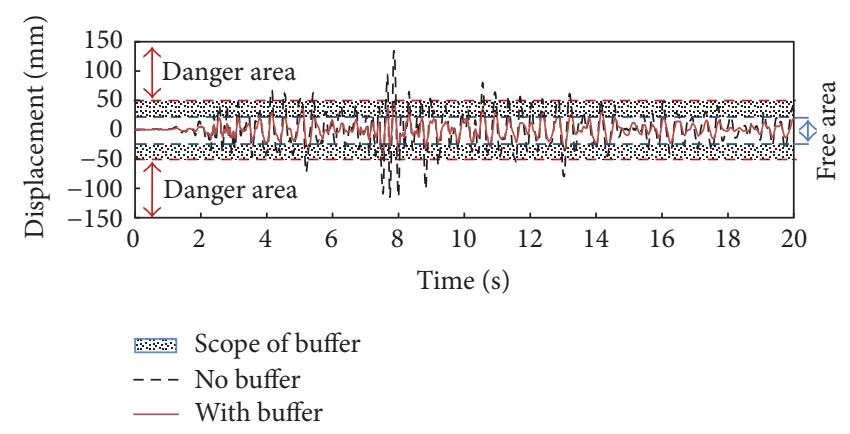

(b) Relative displacement results for a TMD mass with a Fukujima NS seismic wave

FIGURE 14: Amplitude responses for the TMD mass affected by seismic waves: (a) relative displacement result for TMD with a Taft EW seismic wave and (b) relative displacement result for the TMD with a Fukujima NS seismic wave.

Two performance indices are employed (Figure 13). The $J$ values for the displacement response are more than $65.5 \%$, which indicates that the vibration of the platform was significantly improved during the period of earthquake excitation when the bufferable TMD was used. Moreover, analysis of the $\beta$ values shows that a reduction more than $34.5 \%$ was accomplished for the peak displacement response, by applying the bufferable TMD system.

For the acceleration displacement response, the $J$ values are more than $70 \%$, which means that the dynamic performance was considerably improved throughout the entire period of the earthquake. Over the same time, the $\beta$ values are more than $23.8 \%$, which means that the peak response was also decreased by the application of the bufferable TMD system.

\section{Discussion}

The experimental results show that the bufferable damper is effective and can successfully reduce excessive vibration from seismic waves. To further understand the dynamic performances of the bufferable TMD, additional simulation and experimental studies were undertaken to investigate its bufferable, high-response, and absorption characteristics.

5.1. Bufferable Characteristics. Numerical analysis is presented for the displacements of a TMD mass, with and without a buffer, in Figure 14. The free area is defined so that the displacement of the mass block does not exceed its design limitations and collide with the buffers. The TMD then can be dealt with as a traditional mass-spring damper to attenuate 


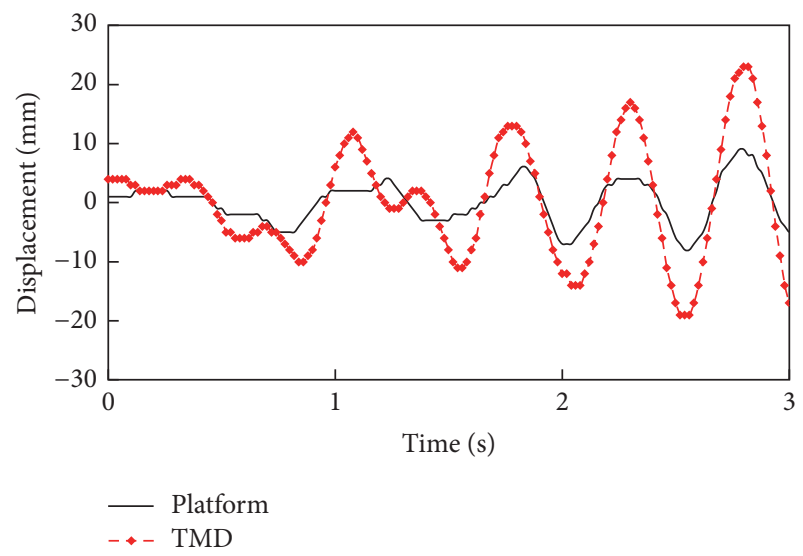

(a) Displacement responses for a Taft EW seismic wave

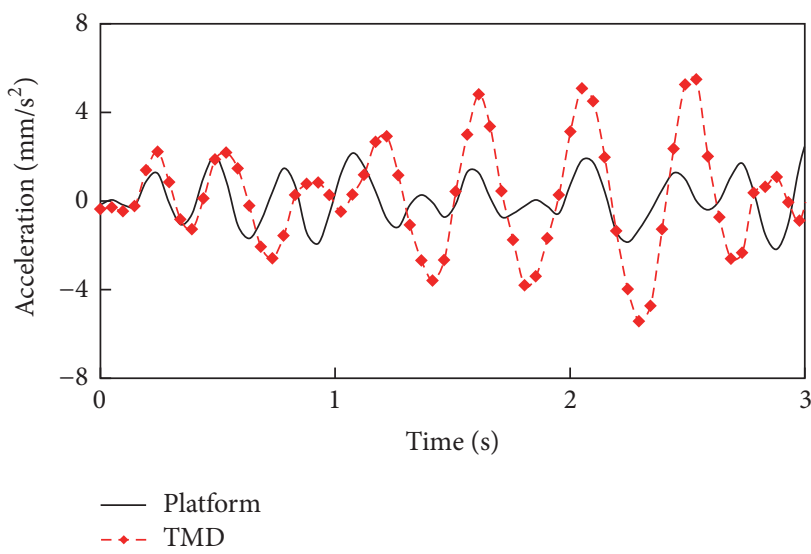

(b) Acceleration responses for a Taft EW seismic wave

FIGURE 15: Experimental responses of the bufferable TMD and platform for the Taft EW seismic waves.

vibrations. The scope of the buffers is presented as horizontal dotted zones in the figures. While the displacement of the mass is within the scope of the buffer, the impact of a mass colliding with the buffers results in an energy dissipation exchange. The danger area is that the displacement of the mass exceeds the frame's limitations. This means that the TMD mass will collide with the frame and that the TMD will be damaged or in some way be made inefficient.

Therefore, in Figure 14(a), related to the relative displacement result of the TMD with a Taft EW seismic wave, it can be seen that the mass of the TMD has exceeded the region of the system, which means that the TMD becomes inefficient during a large earthquake. In Figure 14(b), related to the relative displacement result of the TMD for a Fukujima NS seismic wave, it was found that the buffer can be used to protect the TMD system from damage, and energy is dissipated by buffer.

5.2. High-Response Characteristics. The duration of an earthquake excitation is generally short, and its maximum deforming influence on a platform mainly results from the initial few seconds. Therefore, it is critical that the response of a bufferable TMD occurs rapidly at the initiation of the excitation [23]. By applying the central difference method, the displacements of both a bufferable TMD and an offshore platform during the first $3 \mathrm{~s}$ of excitation can be determined. The initial conditions of main structure can be expressed as

$$
\begin{aligned}
& x_{1}{ }^{(0)}=0, \\
& x_{2}{ }^{(0)}=0, \\
& x_{1}{ }^{(1)}=0, \\
& x_{2}{ }^{(1)}=0 .
\end{aligned}
$$

When the initial conditions from (14) are substituted into (3), the next step differential displacements $x_{1}{ }^{(2)}$ and $x_{2}{ }^{(2)}$ can be obtained:

$$
\begin{aligned}
& x_{1}{ }^{(2)}=\frac{-m_{1} \ddot{x}_{V} \Delta^{2} t}{m_{1}+0.5 c_{1} \Delta t}, \\
& x_{2}{ }^{(2)}=-\ddot{x}_{V} \Delta^{2} t .
\end{aligned}
$$

The ratio of $x_{1}^{(2)}$ and $x_{2}{ }^{(2)}$ can be expressed as

$$
\frac{x_{1}^{(2)}}{x_{2}{ }^{(2)}}=\frac{m_{1}}{m_{1}+0.5 c_{1} \Delta t}<1 \text {. }
$$

Equation (16) shows that, at the initial time, the displacement of the bufferable TMD $x_{2}{ }^{(2)}$ is always greater than the displacement of the offshore platform $x_{1}{ }^{(2)}$; that is, $x_{2}{ }^{(2)}>$ $x_{1}^{(2)}$.

Based on above theoretical analyses in (14)-(16), an experiment was undertaken to investigate this high-response phenomenon. As illustrated in Figure 15, when the offshore platform begins to move as a result of earthquake excitation, the bufferable TMD moves immediately also. In addition, the displacement and acceleration of the bufferable TMD are both considerably greater than those of the offshore platform. This reveals that the bufferable TMD can achieve vibration suppression through its high-response to seismic excitation.

5.3. Absorption Characteristics. To gain a more complete understanding of energy dissipation mechanisms and absorption characteristics of the bufferable damping system, energy dissipation was further examined under the two earthquake-sourced seismic waves. This examination is of particular importance for seismic applications, in which a primary objective of TMD installation should be controlled to avoid damage to the structure of an offshore platform.

Therefore, energy reduction is numerically estimated for the offshore platform equipped with the different TMDs, with 


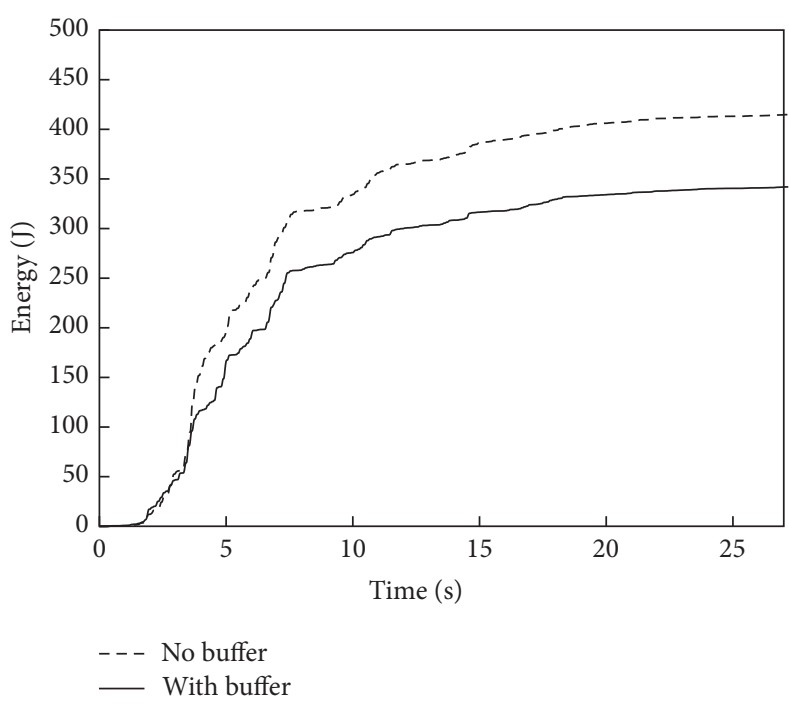

(a) Cumulative energy dissipation with Taft EW seismic waves

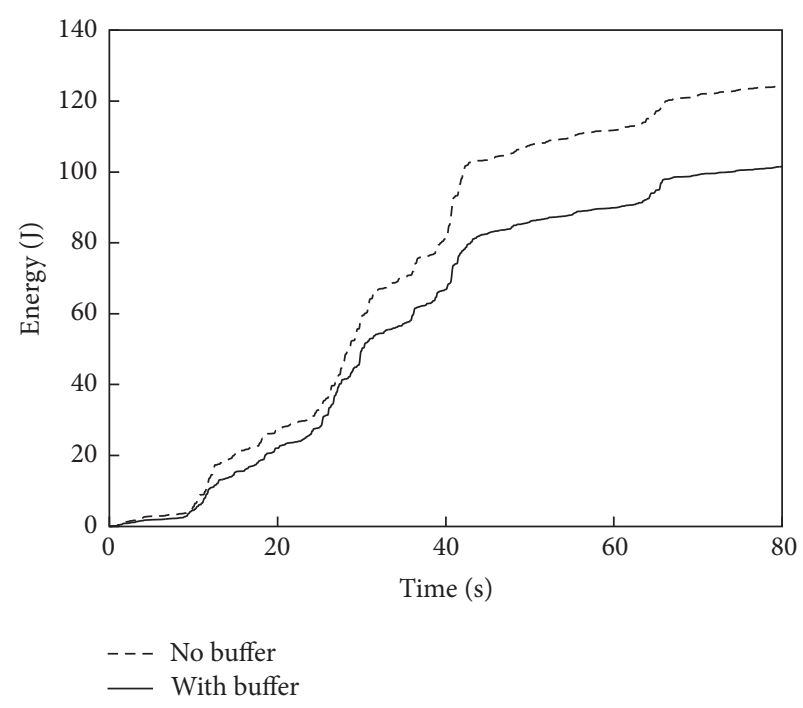

(b) Cumulative energy dissipation with Fukujima NS seismic waves

FIGURE 16: Cumulative energy dissipation with the Taft EW and Fukujima NS seismic waves: (a) displacement results for the Taft EW wave and (b) displacement results for the Fukujima NS wave.

and without a buffer. In this section, a simulation is used to calculate the dissipated energy, while the buffers collided with a mass.

In Figure 16, energy dissipation owing to impact damping is presented for the two seismic excitation cases. Energy reduction at the offshore platform is $17.6 \%$ with the Taft EW seismic waves and $18.4 \%$ under with the Fukujima NS seismic waves. The simulation shows that the impact damping plays significant role, which implies the usefulness of the bufferable TMD even for the offshore platform exposed to large loads of earthquakes.

\section{Conclusions}

This study proposes a bufferable TMD system to mitigate damaging responses of an offshore platform exposed to significant earthquake-sourced seismic waves. A comprehensively numerical and experimental investigation had been conducted to examine bufferable characteristics, high response, and absorption processes of the proposed system.

The main conclusions can be summarized as follows.

(1) A bufferable TMD system was constructed for the experimental study, and the preliminary results indicated that the displacement, acceleration, and frequency performances of the offshore platform were significantly improved under two types of earthquake-induced seismic loads.

(2) Further investigations indicate that the bufferable TMD can effectively absorb the stroke energy by colliding with the buffers on the frame and quickly react during the first 3 seconds of earthquake excitation, which means that the bufferable TMD can effectively decrease the maximum deformation of the structure in the maximum load period of the earthquake excitation.

(3) Both of the numerical and experimental investigations verified that the bufferable TMD constitutes a simple but feasible measure against stroke and response delay problems for vibration suppression under large earthquake loads.

\section{Competing Interests}

The authors declare no conflict of interests regarding the publication of this paper.

\section{References}

[1] B.-L. Zhang and Q.-L. Han, "Network-based modelling and active control for offshore steel jacket platform with TMD mechanisms," Journal of Sound and Vibration, vol. 333, no. 25, pp. 6796-6814, 2014.

[2] B.-L. Zhang, L. Ma, and Q.-L. Han, "Sliding mode $H_{\infty}$ control for offshore steel jacket platforms subject to nonlinear selfexcited wave force and external disturbance," Nonlinear Analysis: Real World Applications, vol. 14, no. 1, pp. 163-178, 2013.

[3] K. Li and A. P. Darby, "An experimental investigation into the use of a buffered impact damper," Journal of Sound and Vibration, vol. 291, no. 3-5, pp. 844-860, 2006.

[4] K. Li and A. P. Darby, "A buffered impact damper for multidegree-of-freedom structural control," Earthquake Engineering and Structural Dynamics, vol. 37, no. 13, pp. 1491-1510, 2008.

[5] C. Salueña, T. Pöschel, and S. E. Esipov, "Dissipative properties of vibrated granular materials," Physical Review E, vol. 59, no. 4, pp. 4422-4425, 1999.

[6] B.-L. Zhang, Q.-L. Han, and X.-M. Zhang, "Event-triggered $H_{\infty}$ reliable control for offshore structures in network environments," Journal of Sound and Vibration, vol. 368, no. 25, pp. 1-21, 2016.

[7] B.-L. Zhang, Q.-L. Han, X.-M. Zhang, and X. Yu, "Sliding mode control with mixed current and delayed states for offshore steel jacket platforms," IEEE Transactions on Control Systems Technology, vol. 22, no. 5, pp. 1769-1783, 2014.

[8] T. Chen, K. Mao, X. Huang, and M. Wang, "Dissipation mechanisms of non-obstructive particle damping using discrete 
element method," Proceedings of SPIE International Symposium on Smart Structures and Materials, vol. 4331, no. 4, pp. 294-301, 2001.

[9] P. A. Cundall and O. D. L. Strack, "A discrete numerical model for granular assemblies," Geotechnique, vol. 29, no. 1, pp. 47-65, 1979.

[10] S. Ekwaro-Osire and I. C. Desen, "Experimental study on an impact vibration absorber," JVC/Journal of Vibration and Control, vol. 7, no. 4, pp. 475-493, 2001.

[11] M. D. Thomas, W. A. Knight, and M. M. Sadek, "The impact damper as a method of improving cantilever boring bars," Journal of Engineering Industry, ASME, vol. 97, no. 3, pp. 859866, 1975.

[12] S. Ema and E. Marui, "A fundamental study on impact dampers," International Journal of Machine Tools and Manufacture, vol. 34, no. 3, pp. 407-421, 1994.

[13] M. Y. Yang, G. A. Lesieutre, S. A. Hambric, and G. H. Koopmann, "Development of a design curve for particle impact dampers," Noise Control Engineering Journal, vol. 53, no. 1, pp. 5-13, 2005.

[14] K. Li and A. P. Darby, "Experiments on the effect of an impact damper on a multiple-degree-of-freedom system," Journal of Vibration and Control, vol. 12, no. 5, pp. 445-464, 2006.

[15] W. Liu, G. R. Tomlinson, and J. A. Rongong, "The dynamic characterisation of disk geometry particle dampers," Journal of Sound and Vibration, vol. 280, no. 3-5, pp. 849-861, 2005.

[16] X. Fang and J. Tang, "Granular damping in forced vibration: qualitative and quantitative analyses," Journal of Vibration and Acoustics, vol. 128, no. 4, pp. 489-500, 2006.

[17] C. J. Wu, W. H. Liao, and M. Y. Wang, "Modeling of granular particle damping using multiphase flow theory of gas-particle," Journal of Vibration and Acoustics, vol. 126, no. 2, pp. 196-201, 2004.

[18] P. B. Zinjade and A. K. Mallik, "Impact damper for controlling friction-driven oscillations," Journal of Sound and Vibration, vol. 306, no. 1-2, pp. 238-251, 2007.

[19] T. Pinkaew, P. Lukkunaprasit, and P. Chatupote, "Seismic effectiveness of tuned mass dampers for damage reduction of structures," Engineering Structures, vol. 25, no. 1, pp. 39-46, 2003.

[20] A. Ghosh and B. Basu, "Effect of soil interaction on the performance of tuned mass dampers for seismic applications," Journal of Sound and Vibration, vol. 274, no. 3-5, pp. 1079-1090, 2004.

[21] C.-C. Lin, J.-M. Ueng, and T.-C. Huang, "Seismic response reduction of irregular buildings using passive tuned mass dampers," Engineering Structures, vol. 22, no. 5, pp. 513-524, 2000.

[22] H.-C. Tsai, "The effect of tuned-mass dampers on the seismic response of base-isolated structures," International Journal of Solids and Structures, vol. 32, no. 8-9, pp. 1195-1210, 1995.

[23] Q. Wu, X. Zhao, R. Zheng, and K. Minagawa, "High response performance of a tuned-mass damper for vibration suppression of offshore platform under earthquake loads," Shock and Vibration, vol. 2016, Article ID 7383679, 11 pages, 2016.

[24] S. S. Rao, Mechanical Vibrations (5th Edition), Prentice Hall, Englewood Cliffs, NJ, USA, 2010. 


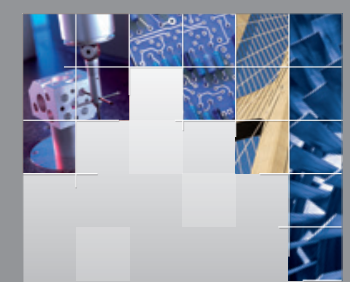

\section{Enfincering}
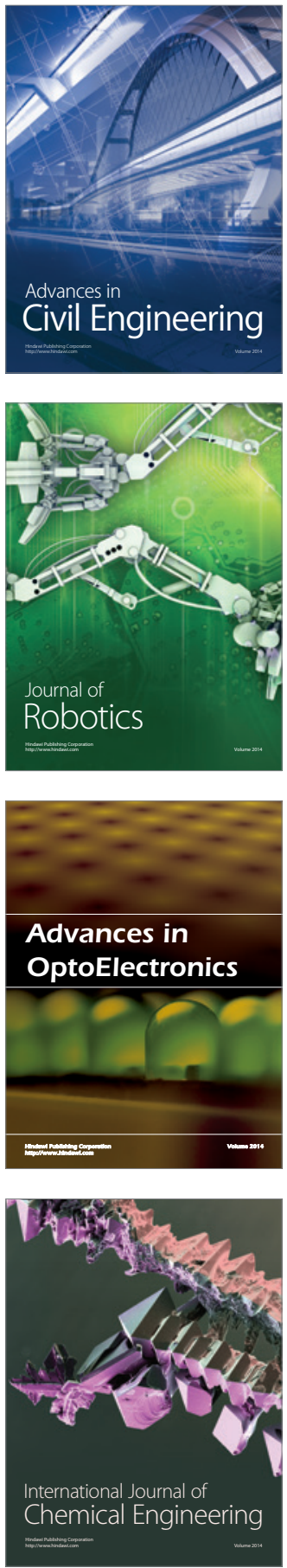

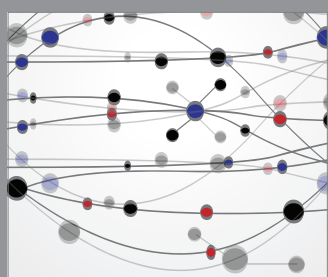

The Scientific World Journal

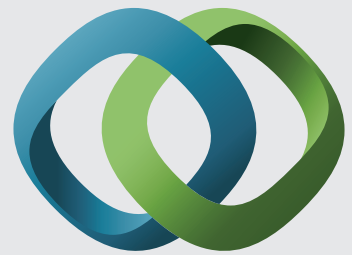

\section{Hindawi}

Submit your manuscripts at

http://www.hindawi.com
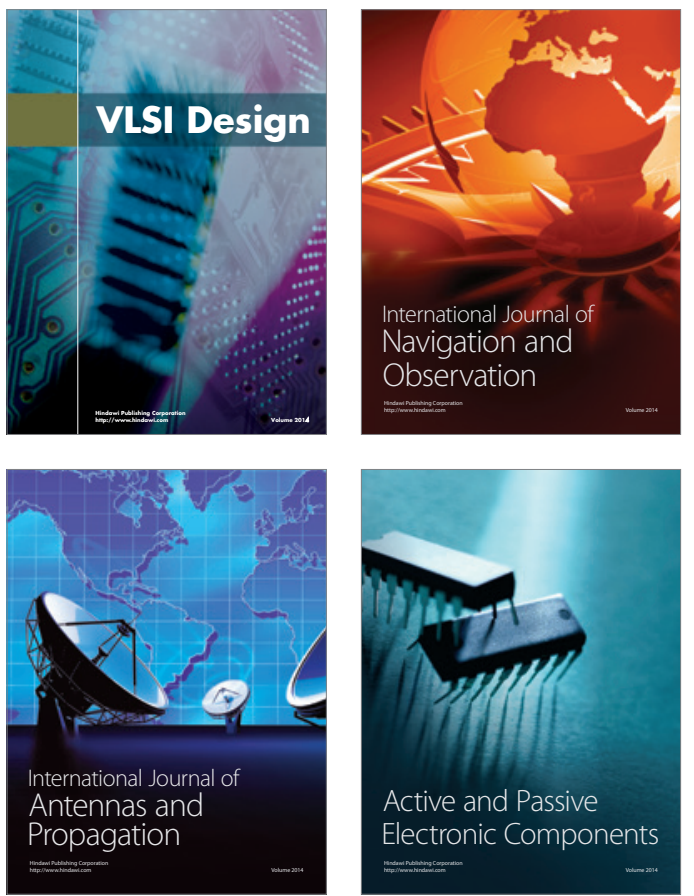
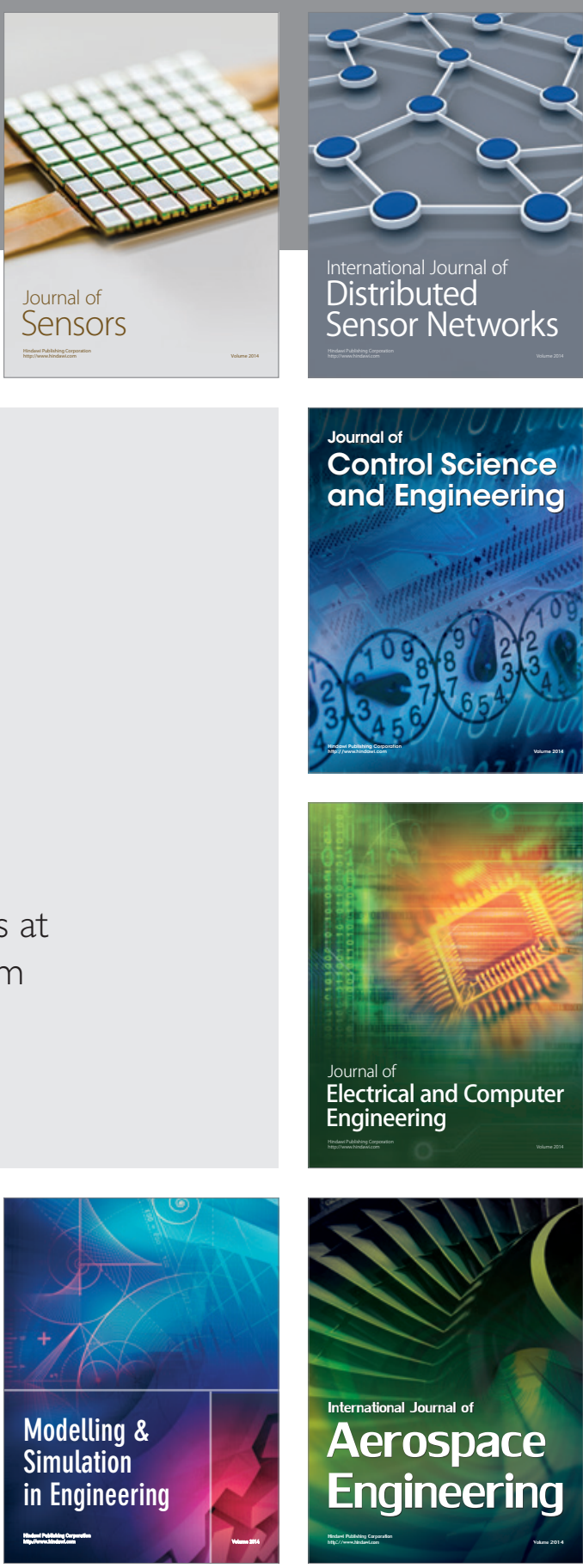

International Journal of

Distributed

Sensor Networks

Journal of

Control Science

and Engineering
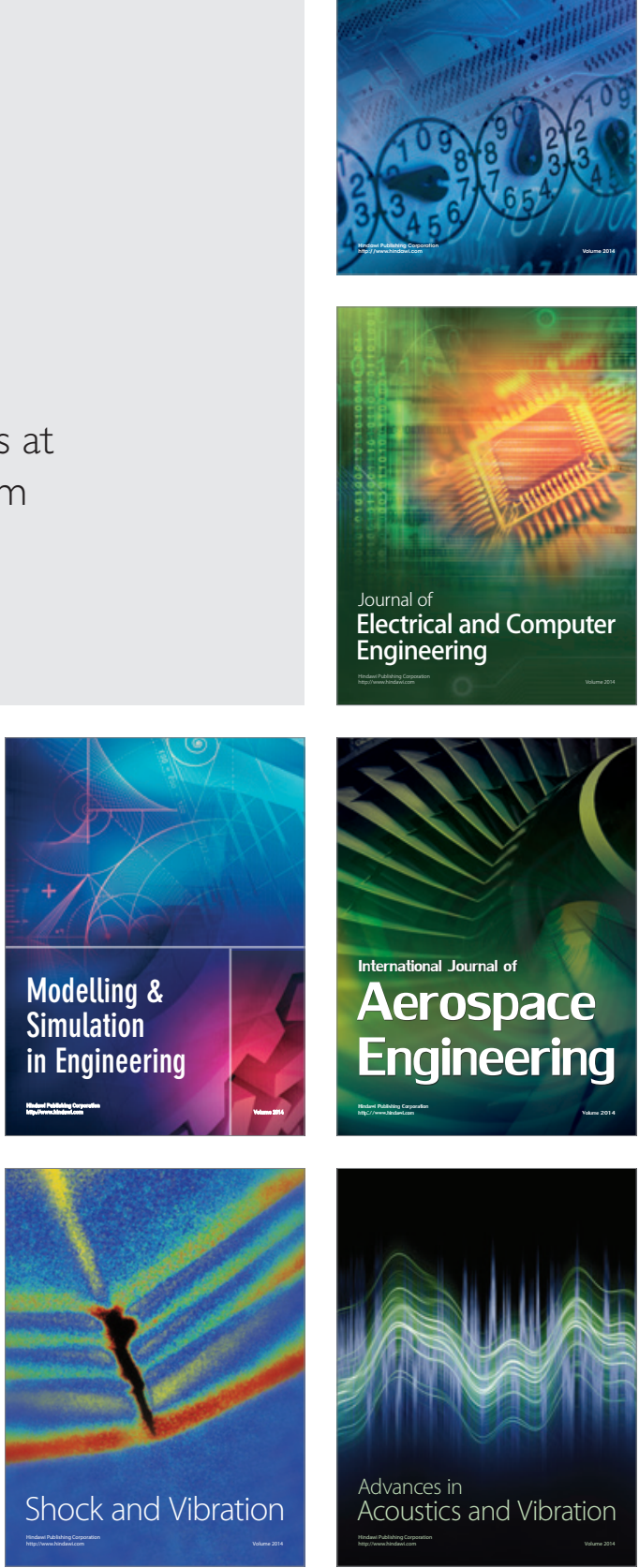